\title{
Bacillus amyloliquefaciens Strain PMB05 Intensifies Plant Immune Responses to Confer Resistance Against Bacterial Wilt of Tomato
}

\author{
Ting-Hsin Ho, ${ }^{1}$ Chiao-Yu Chuang, ${ }^{1}$ Jing-Lin Zheng, ${ }^{1}$ Hong-Hua Chen, ${ }^{1}$ Yu-Shen Liang, ${ }^{2}$ Tzu-Pi Huang, ${ }^{3}$ and \\ Yi-Hsien Lin ${ }^{1, \dagger}$
}

\author{
${ }^{1}$ Department of Plant Medicine, National Pingtung University of Science and Technology, Pingtung, Taiwan \\ 2 Department of Plant Industry, National Pingtung University of Science and Technology, Pingtung, Taiwan \\ ${ }^{3}$ Department of Plant Pathology, National Chung-Hsing University, Taichung, Taiwan \\ Accepted for publication 18 July 2020.
}

ABSTRACT

\begin{abstract}
Tomato is an economic crop worldwide. Many limiting factors reduce the production of tomato, with bacterial wilt caused by Ralstonia solanacearum being the most destructive disease. Our previous study showed that the disease resistance to bacterial soft rot is enhanced by Bacillus amyloliquefaciens strain PMB05. This enhanced resistance is associated with the intensification of pathogen-associated molecular patterns (PAMP)-triggered immunity (PTI). To determine whether the PTI-intensifying Bacillus spp. strains are able to confer disease resistance to bacterial wilt, their effects on PTI signals triggered by PAMP from $R$. solanacearum and on the occurrence of bacterial wilt were assayed. Before assay, a gene that encodes harpin from $R$. solanacearum, PopW, was applied as a PAMP. Results revealed that the B. amyloliquefaciens strain PMB05 was the one strain among 9 Bacillus rhizobacterial strains which could significantly intensify the PopW-induced hypersensitive response (HR) on Arabidopsis leaves.
\end{abstract}

Moreover, we observed that the signals of PopW-induced reactive oxygen species generation and callose deposition were increased, confirming that the PTI was intensified by PMB05. The intensification of the PopW-triggered HR by PMB05 in Arabidopsis was reduced upon treatment with inhibitors in PTI pathways. Furthermore, the application of Bacillus spp. strains on tomato plants showed that only the use of PMB05 resulted in significantly increased resistance to bacterial wilt. Moreover, the PTI signals were also intensified in the tomato leaves. Taken together, we demonstrated that PMB05 is a PTI-intensifying bacterium that confers resistance to tomato bacterial wilt. Screening of plant immunity intensifying rhizobacteria is a possible strategy to control tomato bacterial wilt.

Keywords: Bacillus amyloliquefaciens, bacterial wilt, biological control, genetics and resistance, plant immunity, PopW, tomato
Tomato bacterial wilt caused by Ralstonia solanacearum is known as a devastating disease worldwide. The pathogen infects the roots of plants and causes chlorosis, which leads in turn to wilting and then plant death after establishment (Genin 2010; Hayward 1991; Yabuuchi et al. 1995). $R$. solanacearum is a soilborne pathogen and can survive in the soil for long periods as primary inoculums in various environments (Genin 2010; Grey and Steck 2001; van Elsas et al. 2000). Thus, the bacterial wilt of tomato is very difficult to control in the field. In the past decade, bacterial wilt of tomato and Arabidopsis plants has been controlled to some degree by transferring a protein named plant ferredoxin-like protein (PFLP) from sweet pepper (Huang et al. 2007; Lin et al. 2011, 2010). The PFLP in transgenic plants confers broad-spectrum disease resistance against various bacterial diseases by intensifying defense responses such as pathogen-associated molecular patterns (PAMP)-triggered immunity (PTI) (Ger et al. 2014; Huang et al. 2007; Lin et al. 2010; Namukwaya et al. 2012; Su et al. 2014; Tang et al. 2001; Yip et al. 2011). PAMP-triggered immunity involves the

${ }^{\dagger}$ Corresponding author: Y.-H. Lin; yhlin@mail.npust.edu.tw

Funding: This work was supported by grants to Y.-H. Lin from the Ministry of Science and Technology, Taiwan (MOST 105-2628-B-020-002-MY3 and MOST 108-2628-B-020-001)

*The $e$-Xtra logo stands for "electronic extra" and indicates that one supplementary figure is published online.

The author(s) declare no conflict of interest. activation of a defense pathway commonly initiated by the recognition of PAMPs such as flagellin, peptidoglycan, elongation factor, and harpin on plant cell surfaces (Bigeard et al. 2015; Dodds and Rathjen 2010; Gust et al. 2007; Oh and Beer 2005; Postel and Kemmerling 2009). For example, in Arabidopsis thaliana, the bacterial flg22 peptide specifically binds to the flagellin-sensing2 (FLS2) receptor to activate defense pathways such as those involving $\mathrm{Ca}^{2+}$ influx, rapid reactive oxygen species (ROS) generation, and the further expression of defense genes (Ali and Reddy 2008; Boller and Felix 2009; Boudsocq et al. 2010; Chinchilla et al. 2006; Monaghan and Zipfel 2012). In addition, harpin is regarded as a kind of PAMPs, but it can induce the typical effector-triggered immunity (ETI) responses such as hypersensitive response (HR) necrosis and cytoskeleton disruption (Guan et al. 2013). And, harpin is different from general effector secreted by pathogenic bacteria. The extracellular harpins might bind to HrpNinteracting protein in the apoplasts of plant tissues (Oh and Beer 2005). Relatedly, due to the similar characteristics of different harpins, disease resistance is enhanced by treating these proteins. For example, disease resistance against Tobacco mosaic virus and abiotic stresses induced by another harpin encoded by pop $W$ from Ralstonia solanacearum, has also been demonstrated ( $\mathrm{Li}$ et al. 2011; Liu et al. 2016). Therefore, flg22 and harpin can be applied as PAMPs to mimic the immune response initiated on the cell surface of a host plant during pathogen attack.

Recently, the use of antagonistic microorganisms has been considered one of the most promising approaches for controlling tomato bacterial wilt due to their antimicrobial compounds production, induced resistance, and aggressive colonization of rhizosphere (Huang et al. 2016; Kwon and Kim 2014; Ramadasappa et al. 2012; Song et al. 2018; Tahir et al. 2017; Yim et al. 2013). 
Among them, many strains of Bacillus species are used due to their broad-spectrum antagonistic effect against pathogens (Kumar et al. 2012; Romero et al. 2007; Zalila-Kolsi et al. 2016). In addition, the endospore formation of Bacillus spp. is advantageous for their application due to the tolerance against adverse environments and long-term storage capacity it imparts (de Boer and Diderichsen 1991). Besides that, our own previous study demonstrated that Bacillus amyloliquefaciens strain PMB05 is the only strain capable of intensifying the PTI signals triggered by PAMP from soft rot bacteria and that it further confers resistance to bacterial soft rot disease (Wang et al. 2019).

In this study, we sought to determine the effects of strains of Bacillus species on the PTI signals activated by a PAMP from $R$. solanacearum and their contribution to disease resistance against bacterial wilt disease. Firstly, the PopW from the R. solanacearum strain Ps152 was cloned and expressed as a PAMP elicitor. The effects of nine Bacillus spp. strains isolated from rhizosphere on PopW-mediated HR, rapid ROS generation, and callose deposition in Arabidopsis plants were then assayed. Next, the PTI pathways of PopW-mediated HR intensified by PMB05 were confirmed. Furthermore, their efficacies in terms of increasing the disease resistance of tomato to bacterial wilt were evaluated. The associated results provide evidence that the improvement of disease resistance to bacterial wilt in tomato was associated with the activation of PTI.

\section{MATERIALS AND METHODS}

Plants and bacteria growth conditions. Arabidopsis thaliana ecotype Columbia (Col-0) and tomato (Solanum lycopersicum 'Known You 301') (Known-You Seed, Taiwan) seeds were sown in peat moss and allowed to grow for 1 week. The germinated seedlings were then transferred to individual pots for another 2 weeks. The 3-week-old plants were then used in further experiments, including a HR assay, plant defense signals assay, and biocontrol efficacy assay. All the plants were grown in a growth chamber (Hipoint, Taiwan) at $28^{\circ} \mathrm{C}(16 \mathrm{~h}$ light $/ 8 \mathrm{~h}$ dark).

Bacterial wilt pathogen from tomato, $R$. solanacearum strain Ps152, was incubated on an 2,3,5-triphenyltetrazolium chloride (TZC) agar plate at $28^{\circ} \mathrm{C}$ for $48 \mathrm{~h}$ (Kelman 1954; Lin et al. 2010). For protein expression, Escherichia coli DH5 $\alpha$ and BL21 (DE3) were grown in Luria-Bertani (LB) broth or on LB agar plates with ampicillin at $100 \mu \mathrm{g} / \mathrm{ml}$ at $37^{\circ} \mathrm{C}$. Nine Bacillus spp. strains were isolated from a soil sample from an experimental farm of the Department of Plant Medicine (PMB05) or from soil samples from Daren Forest of National Pingtung University of Science and Technology (DR237, DR238, DR242, DR243, DR244, DR258, DR301, and DR303). All the Bacillus spp. strains were incubated in nutrient agar plates at $28^{\circ} \mathrm{C}$ for $24 \mathrm{~h}$ before each assay.

Plasmid construct and expression of PopW. To express the PopW from $R$. solanacearum strain Ps152, specific primers (PopWNdeI-F: 5'-CATATGTCCATCCAGATTGATCG-3', and PopWBamHI-R: 5'-GGATCCTCAGCCCGAGTAGGCCTT-3') were designed according to the sequence of the pop $W$ gene (accession number EF080949) from strain ZJ3721 (Li et al. 2010). The amplicon was cloned in an expression vector, pET16b (Novagen, U.S.A.), which can express the recombinant protein with a $6 \times$ His tag at the N-terminal. The coding sequence of the pop $W$ gene was in-frame constructed in $\mathrm{pET} 16 \mathrm{~b}$ to generate $\mathrm{pET} 16 \mathrm{~b}-\mathrm{PopW}$. Then, the pET16b-PopW and pET16b (vector alone) were transformed into Escherichia coli BL21 (DE3) to obtain the transformants OEPopW and 16B, respectively. To express the PopW, OE-PopW was cultured in LB broth with $1 \mathrm{mM}$ of isopropylthio- $\beta$-D-galactoside at $37^{\circ} \mathrm{C}$ for $8 \mathrm{~h}$. The cells of OE-PopW were then collected and sonicated in $25 \mathrm{mM}$ Tris- $\mathrm{HCl}(\mathrm{pH} 7.0)$ for $1 \mathrm{~h}$ to obtain the sonicated extract. The sonicated extract was incubated at $100^{\circ} \mathrm{C}$ for $10 \mathrm{~min}$, followed by centrifugation at $12,000 \times g$ for 20 min to obtain the protein extract. To confirm that the PopW was expressed in OEPopW, $1.0 \mu \mathrm{g}$ of protein extract was fractionated in $15 \%$ sodium dodecyl sulfate-polyacrylamide gel electrophoresis and transferred to an Immun-Blot PVDF membrane (Bio-Rad, U.S.A.). The membrane was reacted with Anti-6x-His rabbit polyclonal antiserum (Rockland, U.S.A.) and HRP-conjugated goat antirabbit IgG (Millipore, U.S.A.) sequentially. Then, the membrane was stained in a Metal Enhanced DAB Substrate Kit (Thermo, U.S.A.) according to the manufacturer's procedures.

HR ratio assay on $A$. thaliana. To assess whether the HR could be induced by PopW, leaf infiltration of plants was carried out with the protein extract at $0.5,1.0,1.5$, and $2.0 \mathrm{mg} / \mathrm{ml}$. The HR ratio was calculated as (HR necrosis area/infiltration area) $\times 100 \%$. The HR induction assay of the Arabidopsis leaves was performed according to a previously established method (Lin et al. 2011; Lin et al. 2010; Su et al. 2014). To assay the effect of Bacillus spp. strains on the occurrence of HR, coinfiltration of the protein extract and bacterial suspensions was performed. Before infiltration, each bacterial strain was incubated in nutrient broth at $28^{\circ} \mathrm{C}$ for $24 \mathrm{~h}$, followed by centrifugation at $12,000 \times g$ for $10 \mathrm{~min}$ to obtain the pellet. The pellet was suspended in sterilized distilled water, and its $\mathrm{OD}_{600}$ was adjusted to 0.3 as the bacterial suspension. Each bacterial suspension and protein extract at $1.0 \mathrm{mg} / \mathrm{ml}$ was mixed with an equal volume. Thirty repeats of each treatment were analyzed in one individual experiment.

Observation of callose deposition. To observe the effect of Bacillus spp. strains on PopW-triggered callose deposition, the leaves of A. thaliana Col-0 were infiltrated with the mixture containing the protein extract of PopW and the bacterial suspension of each Bacillus sp. strain. The infiltrated leaf samples were collected for callose staining at $8 \mathrm{~h}$ postinfiltration (Su et al. 2014). Briefly, the leaves were cut in tiny strips and washed with $95 \%$ ethanol for $2 \mathrm{~h}$ and then washed in $0.1 \mathrm{M}$ phosphate buffer ( $\mathrm{pH} 8.0$ ). The leaf strips were further incubated with $0.01 \%$ aniline-blue (Sigma, U.S.A.) in $0.1 \mathrm{M}$ phosphate buffer for $2 \mathrm{~h}$ for staining before observation under a fluorescence microscope with an excitation/ emission at 340 to $380 / 400$ to $425 \mathrm{~nm}$ filter set (Leica, Germany). The fluorescent strength of callose deposition of each treatment was assayed by the Image $\mathrm{J}$ software under a consistent threshold (Rasband 1997-2016). Each value was collected from 10 samples of individual leaves in each treatment as repeats.

Observation of rapid $\mathrm{H}_{2} \mathrm{O}_{2}$ generation. To assay the effect of Bacillus strains on PopW-induced rapid $\mathrm{H}_{2} \mathrm{O}_{2}$ generation, an observation was performed at $1 \mathrm{~h}$ postinfiltration with the mixture containing the protein extract of PopW and the bacterial suspension of each Bacillus sp. strain. Leaf strips of A. thaliana Col-0 were stained with $20 \mu \mathrm{M}$ of 2',7'-dichlorodihydrofluorescein diacetate (Molecular Probes, U.S.A.) in 0.1 M phosphate buffer ( $\mathrm{pH}$ 8.0) in the dark for $15 \mathrm{~min}$. Then, the stained leaf strips were washed with PBS buffer and were further observed under a fluorescence microscope with an excitation/emission at 465 to $495 / 515$ to $555 \mathrm{~nm}$ filter set (Leica, Germany). The strength of $\mathrm{H}_{2} \mathrm{O}_{2}$ generation was assayed by the Image $\mathbf{J}$ software to measure the fluorescent intensity under a consistent threshold (Rasband 1997-2016). Each value was collected from 10 samples of individual leaves in each treatment as repeats.

Inhibition of HR through plant defense pathway. Both the activation of NADPH oxidase and calcium influx are early hallmarks in PTI signaling (Torres 2010). In order to determine whether the Bacillus-intensified HR was associated with the PTI, the reduction of the HR ratio on Arabidopsis thaliana Col-0 was assayed upon the inhibition of calcium influx and NADPH oxidase. The inhibition of NADPH oxidase and calcium influx was performed by using diphenyleneiodonium chloride (DPI) and lanthanum chloride $\left(\mathrm{LaCl}_{3}\right)$, respectively ( $\mathrm{Su}$ et al. 2014). The infiltration was carried out with mixtures containing the PopW and the bacterial suspension of each Bacillus sp. strain with $\mathrm{LaCl}_{3}$ or DPI at 40 or $80 \mu \mathrm{M}$ of the final concentrations. The HR ratio was 
calculated at $24 \mathrm{~h}$ postinfiltration. Ten repeats were performed in each treatment in this assay.

Disease severity assay on tomato. To evaluate the efficacy with which tomato bacterial wilt disease was reduced by Bacillus strains, diseased soil was utilized. Specifically, the bacterial cells of $R$. solanacearum strain Ps152 were washed from the TZC medium with sterilized distilled water and the bacterial suspension was adjusted to $\mathrm{OD}_{600} 0.3$ to serve as the inoculum. The inoculum was mixed with a 10 -fold volume of peat moss to prepare the diseased soil. Before inoculation, 12 3-week-old tomato plants were soaked in a bacterial suspension of each Bacillus sp. strain for $30 \mathrm{~min}$. For inoculation, the treated plants were then transplanted into the diseased soil 2 days later. At 10 days postinoculation, the index of wilting symptoms was rated from 0 to 4 ( 0 , no wilting; 1 , one leaf wilting; 2, two leaves wilting; 3 , three leaves wilting; and 4, plant death), and then the total number $(n=10)$ of plants with different levels of wilting symptoms was counted. The disease severity of each treatment was calculated using the following formula: $\left[\left(0 \times \mathrm{N}_{0}\right.\right.$ $\left.\left.+1 \times \mathrm{N}_{1}+2 \times \mathrm{N}_{2}+3 \times \mathrm{N}_{3}+4 \times \mathrm{N}_{4}\right) /(4 \times \mathrm{N})\right] \times 100 \%$ (Winstead and Kelman 1952). Four sets of each treatment were carried out as repeats in one experiment.

Intensification of PAMP-mediated ROS generation and callose deposition by $B$. amyloliquefaciens strain PMB05 on tomato. To observe the effect of $B$. amyloliquefaciens strain PMB05 on PAPM-triggered callose deposition and ROS generation, tomato leaves were infiltrated as described above. Besides PopW, flg22 Rs (QRLSTGMRVNSAQDDAAAYASA) from $R$. solanacearum (Hong et al. 2018) was synthesized by LifeTein LLC (South Plainfield, U.S.A.). The synthesized flg $22_{\mathrm{Rs}}$ was prepared in $25 \mathrm{mM}$ Tris- $\mathrm{HCl}$ buffer ( $\mathrm{pH} 7.5$ ) at $5 \mathrm{mM}$ before assay, and the final concentration was $0.5 \mu \mathrm{M}$ in each treatment. The infiltrated tomato leaves were shaved and then cut into tiny strips before undergoing further staining. The strength of fluorescent signals was assayed by the Image $\mathrm{J}$ software to measure the intensities under a consistent threshold (Rasband 1997-2016). Each value was collected from 10 samples subjected to each treatment as repeats.

Inhibitory effect of Bacillus spp. against $R$. solanacearum. The inhibitory activity assay was performed on a nutrient agar plate. A single colony of each Bacillus sp. strain was spotted on the surface of a plate with sterilized toothpicks and further incubated in $28^{\circ} \mathrm{C}$ for $24 \mathrm{~h}$. Then, a bacterial suspension of $R$. solanacearum strain Ps152 was prepared and sprayed on the plates. After $48 \mathrm{~h}$ incubation, the inhibitory zone was measured. The inhibitory zone of each Bacillus sp. strain to Ps152 was determined with 20 repeats.

Statistical analysis. Statistical analysis was performed with the IBM SPSS Statistics software for Windows, version 25 (IBM Corp., U.S.A.). Analysis of variance was used to test for differences between treatments in all experiments. Post hoc tests (Tukey's honestly significant difference test) were used to compare means between treatments to obtain $F$ and $P$ values.

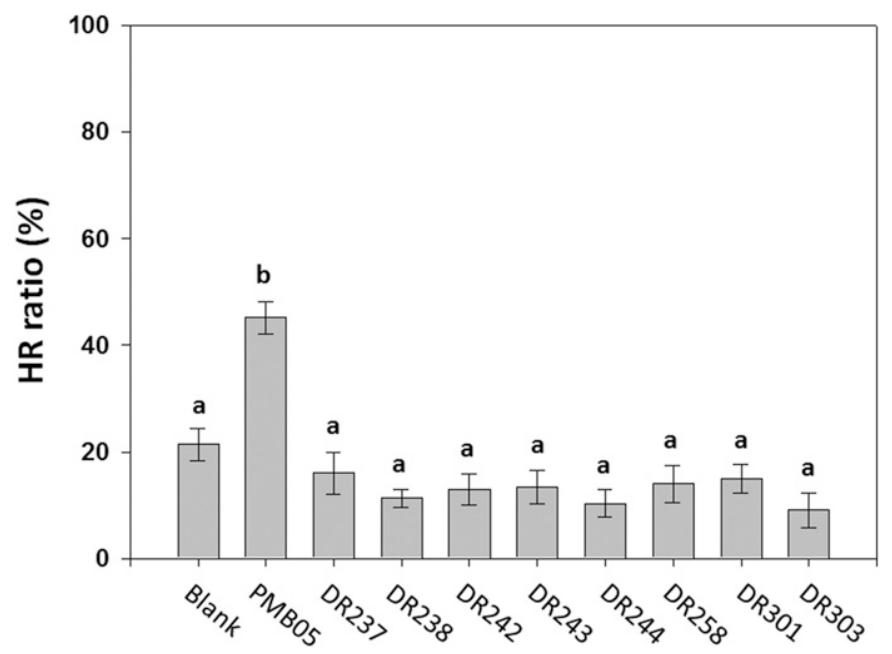

Fig. 2. PopW-induced hypersensitive response (HR) ratio intensified by Bacillus spp. on the leaves of Arabidopsis thaliana. The assay was performed with the mixtures of PopW and bacterial suspensions of distinct Bacillus spp. strains at $0.5 \mathrm{mg} / \mathrm{ml}$ and final concentrations of $10^{8} \mathrm{CFU} / \mathrm{ml}$. Blank indicates that the treatment consisted of sterilized water only. The ratio of HR necrosis in three infiltrated leaves of each plant was calculated at $24 \mathrm{~h}$ postinfiltration. Thirty plants were used in each treatment as repeats. Different letters above the columns indicate significant differences between the treatments based on Tukey's honestly significant difference test $(F=$ 11.44; $P<0.05)$.
A
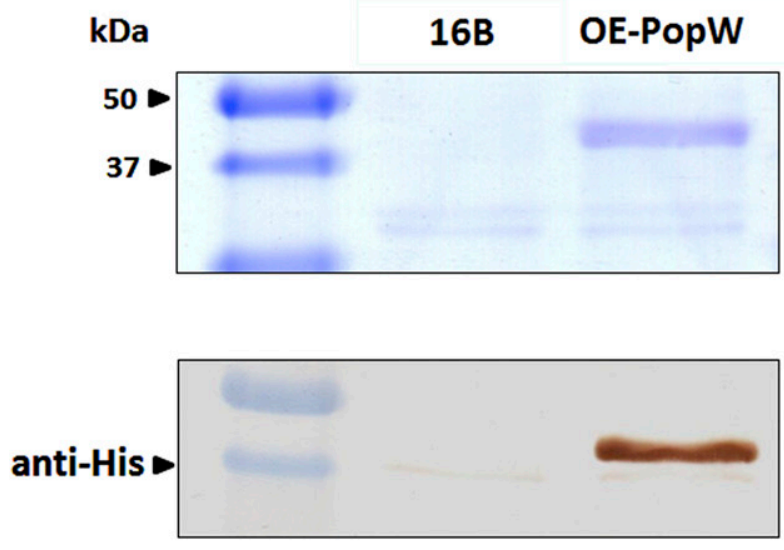

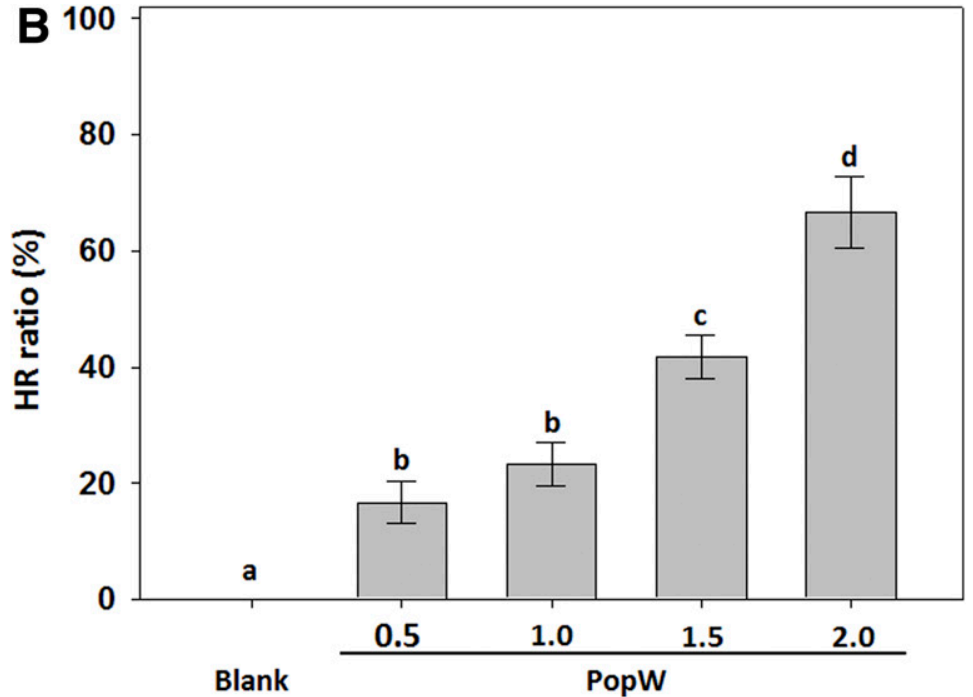

Blank

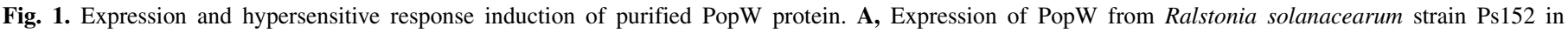

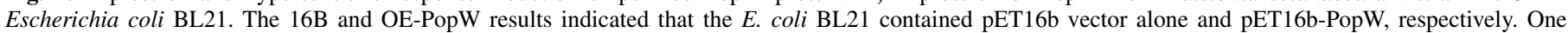

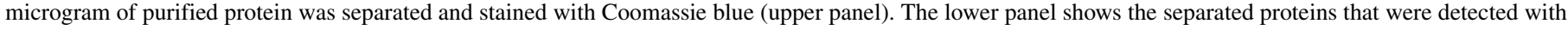

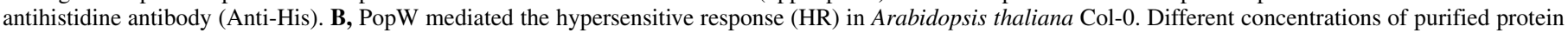

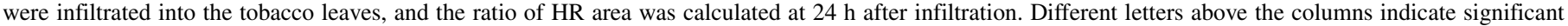
differences based on Tukey's honestly significant difference test $(F=42.34 ; P<0.05)$. 


\section{RESULTS}

PopW-mediated HR on A. thaliana. In order to use a PAMP from $R$. solanacearum as an elicitor, the pop $W$ gene, which encodes a harpin was amplified from strain Ps152. The DNA sequence of the popW gene from Ps 152 was $99 \%$ identical to that from strain ZJ3721 (EF080949). The coding sequence of the popW gene was constructed into pET16b and then transformed into E. coli BL21 (DE3) to obtain OE-PopW. The OE-PopW was further used to express PopW. The signals of PopW in SDS-PAGE and in western blot detected with anti-6× His antiserum were visualized at around $40 \mathrm{kDa}$ (Fig. 1A). The protein extract was then used to induce HR necrosis in Arabidopsis leaves. The results revealed that the HR was induced by the protein extract of PopW at all concentrations. The HR ratios for Arabidopsis leaves produced with 0.5, 1.0, 1.5, and $2.0 \mathrm{mg} / \mathrm{ml}$ of PopW protein were 22.8, 41.9, 56.3, and 69.6\%, respectively (Fig. 1B).

A

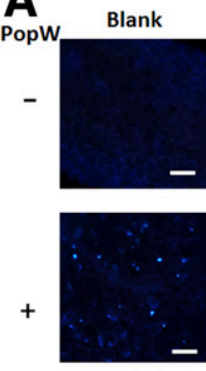

DR243
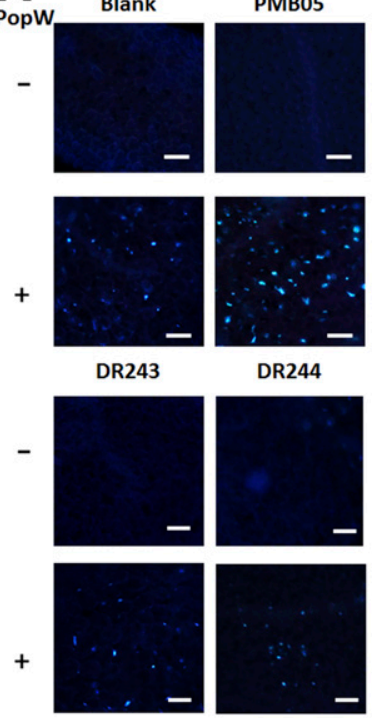

DR244

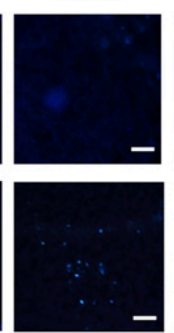

B

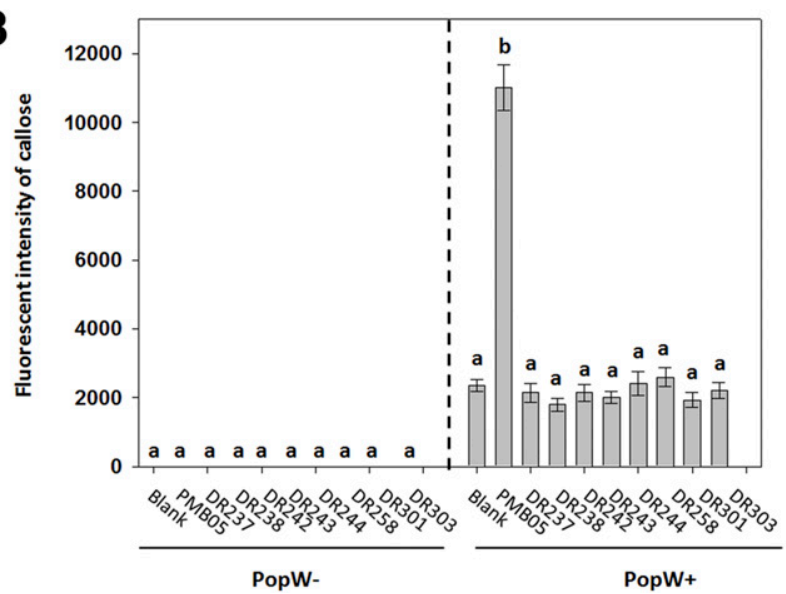

Fig. 3. Effects of Bacillus spp. strains on callose deposition upon PopW treatment in Arabidopsis thaliana. A, Results of the callose deposition induced by the infiltration with PopW alone or the mixtures of PopW and distinct Bacillus spp. strains in Arabidopsis plants. To observe the callose deposition, the infiltrated leaves were collected and stained with $0.01 \%$ aniline blue at $8 \mathrm{~h}$ postinfiltration. The scale bars indicate $50 \mu \mathrm{m}$ in length. Blank indicates that the treatment consisted of sterilized water only. The symbols "+" and "-" indicate the inclusion and exclusion, respectively, of PopW in the treatments. B, The fluorescent intensities of the callose count as determined by ImageJ. Different letters above the columns indicate significant differences according to Tukey's honestly significant difference test $(F=69.01 ; P<0.05)$.
Intensification of PopW-induced HR by Bacillus spp. on A. thaliana. Before the experiment, we confirmed that all of the bacterial suspensions of Bacillus spp. strains did not induce HR in the leaves of $A$. thaliana Col-0 plants. To evaluate the intensification of PopW-induced HR by Bacillus spp. strains, the coinfiltration of the PopW protein and bacterial suspension of each Bacillus sp. was performed in the assay. The results indicated that none of the bacterial strains increased the PopW-induced HR ratio significantly, except strain PMB05 (Fig. 2). The PMB05 strain increased the HR ratio to $45.2 \%$, a significant increase from $21.4 \%$ in the treatment with PopW alone. The ratios yielded by the treatments with DR237, DR238, DR242, DR243, DR244, DR258, DR301, and DR303 were 16.1, 11.3, 12.9, 13.5, 10.4, 14.1, 14.9, and $9.1 \%$, respectively.

Intensification of PopW-mediated callose deposition by Bacillus spp. strains. To assess the association between increasing HR and PTI activation, the intensification of PopW-

A

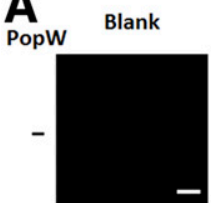

PMB05

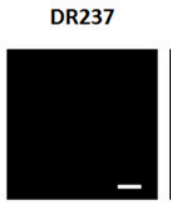

DR238

DR242
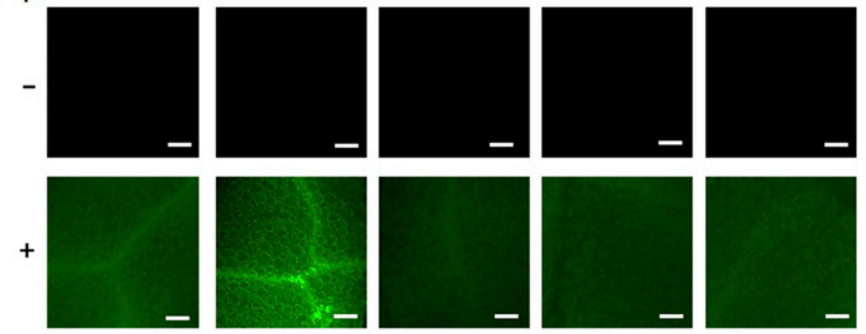

DR243

DR244

DR258

DR301

DR303
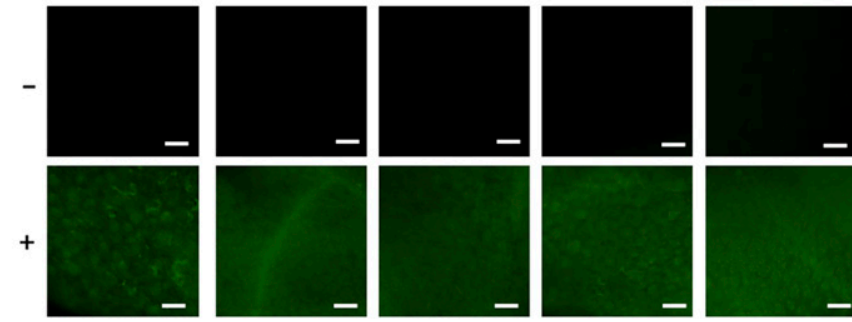

B

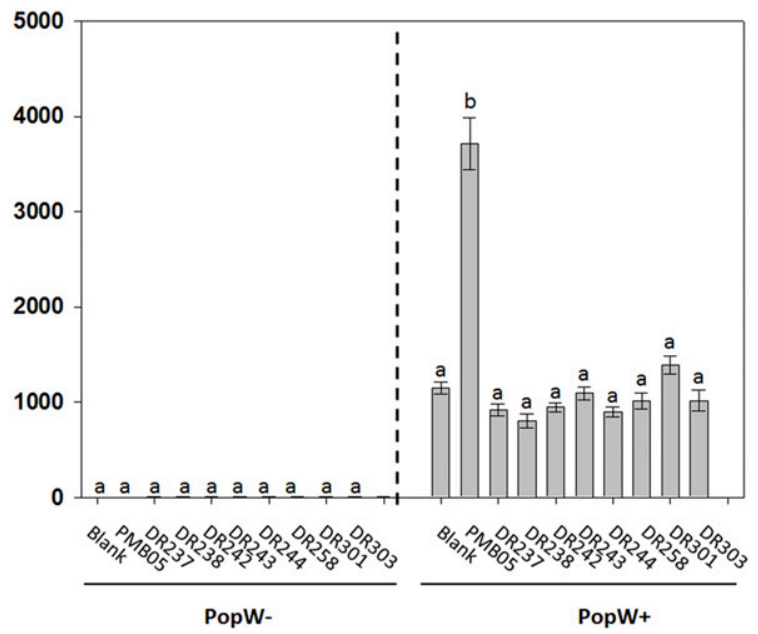

Fig. 4. Effects of Bacillus spp. strains on rapid reactive oxygen species (ROS) generation upon PopW treatment in Arabidopsis thaliana. A, Rapid $\mathrm{H}_{2} \mathrm{O}_{2}$ generation induced by PopW alone or the mixtures of PopW and distinct Bacillus spp. strains in Arabidopsis plants. To observe the rapid ROS generation, the infiltrated leaves were collected and stained with $20 \mu \mathrm{M}$ of $\mathrm{H}_{2}$ DCFDA at $1 \mathrm{~h}$ postinfiltration. The scale bars indicate $50 \mu \mathrm{m}$ in length. Blank indicates that the treatment consisted of sterilized water only. The symbols "+" and "-" indicate the inclusion and exclusion, respectively, of PopW in the treatments. B, Fluorescent intensities of the ROS count as determined by ImageJ. Different letters above the columns indicate significant differences according to Tukey's honestly significant difference test $(F=$ 42.11; $P<0.05)$. 
mediated callose deposition and $\mathrm{H}_{2} \mathrm{O}_{2}$ generation by PMB05 was assayed on Arabidopsis plants. The fluorescent signals indicated that callose deposition was induced by PopW around the cells of Arabidopsis leaves. However, no signal was observed in the treatment with PMB05, DR237, DR238, DR242, DR243, DR244, DR258, DR301, or DR303 alone. Moreover, both the quantity and strength of the PopW-induced callose deposition were only intensified by strain PMB05. However, the assay showed no visible increase in the signals in the treatments with other Bacillus spp. strains (Fig. 3A). Compared with the treatment with PopW alone, the intensity in the PopW treatment with PMB05 was significantly increased to $467.4 \%$, whereas the intensities with DR237, DR238, DR242, DR243, DR244, DR258, DR301, and DR303 were 90.9, 76.5, 91.0, 85.4, 102.1, 110.4, 81.8, and $93.5 \%$, respectively (Fig. 3B).

Intensification of PopW-mediated ROS generation by Bacillus spp. strains. The intensification of PopW-mediated rapid ROS generation by Bacillus spp. strains was observed at $1 \mathrm{~h}$ postinfiltration. The results revealed that the fluorescent signals of ROS were induced by PopW in Arabidopsis plants. However, weak signals or no signals were observed in the treatment with each Bacillus sp. strain alone. In addition, the ROS signals were stronger in the treatment with PMB05 than in those with DR237, DR238, DR242, DR243, DR244, DR258, DR301, or DR303 (Fig. 4A). Compared with the treatment with PopW alone, the intensity in the PopW treatment with PMB05 was significantly increased to $323.8 \%$. Meanwhile, the intensities in the treatments with DR237, DR238, DR242, DR243, DR244, DR258, DR301, and DR303 were $80.1,70.4,82.4,95.2,78.3,87.9,121.0$, and $88.4 \%$, respectively (Fig. 4B).

Inhibition of plant defense pathway. To provide more details regarding the effect of B. amyloliquefaciens PMB05 in intensifying PopW-mediated defense responses, the roles of the calcium channel and NADPH oxidase in A. thaliana were investigated. The results revealed that the PopW-mediated HR was intensified by PMB05 to 50.2\% compared with $23.9 \%$ with the blank treatment (Fig. 5A). Moreover, this intensification of the HR was reduced by 40 and $80 \mu \mathrm{M}$ of DPI to 37.2 and $23.0 \%$, respectively, from $47.5 \%$ at $0 \mu \mathrm{M}$ (Fig. 5B). Similarly, this intensification of the HR was inhibited by 40 and $80 \mu \mathrm{M}$ of $\mathrm{LaCl}_{3}$ to 39.2 and $28.2 \%$, respectively, from $48.5 \%$ at $0 \mu \mathrm{M}$ (Fig. 5C).

Disease resistance intensified by Bacillus $\mathrm{sp}$. To assess whether the resistance against bacterial wilt was improved by strain
PMB05, the disease severity on tomato caused by $R$. solanacearum Ps152 was assayed. In terms of the wilt symptoms that appear on tomato plants, most plants treated with PMB05 were symptomless at 10 days postinoculation (Fig. 6A). The results revealed that the disease severity following PMB05 treatment $(36.7 \%)$ was lower than that following DR237 (73.5\%), DR238 (56.8\%), DR242 (54.2\%), DR243 (59.1\%), DR244 (62.5\%), DR258 (83.7\%), DR301 $(72.4 \%)$, DR303 $(68.9 \%)$, and control treatment $(86.6 \%)$ at 1 week postinoculation (Fig. 6A).

Intensification of PAMP-mediated ROS generation and callose deposition by $B$. amyloliquefaciens strain PMB05 on tomato. To connect the intensification of PTI by B. amyloliquefaciens strain PMB05 with the disease resistance to bacterial wilt of tomato, the effects of PMB05 on PAMP-mediated ROS generation and callose deposition were assayed on tomato leaves. The results revealed that the callose deposition was triggered by both flg $22_{\mathrm{Rs}}$ and PopW, and that the fluorescent signals were stronger due to the treatment with PMB05. Compared with the treatments with flg $22_{\mathrm{Rs}}$ and PopW alone, the intensities were significantly increased to 545.5 and $1,026.1 \%$, respectively (Fig. 7A). Similarly, the fluorescent signals of rapid ROS generation triggered by flg $22_{\mathrm{Rs}}$ and PopW were increased by the treatment with PMB05. Compared with the treatments with flg $22_{\mathrm{Rs}}$ and PopW alone, the intensities were significantly increased to 320.1 and $251.1 \%$, respectively (Fig. 7B). In the analyses of both callose deposition and rapid ROS generation, the PMB05 did not induce the production of fluorescent signals as these result in a blank treatment.

Inhibitory effect of Bacillus spp. against $R$. solanacearum. To understand whether the control effect of Bacillus spp. on bacterial wilt is related to the inhibitory effect, an antagonistic assay was carried out on nutrient agar plates. The results revealed that the growth of $R$. solanacearum was inhibited by all the tested Bacillus spp. strains. The inhibition was divided into three groups: inhibitory index 1 indicated that weak inhibitory zones were induced by the Bacillus spp. strains, with the strains with this index value including PMB05, DR237, DR258, and DR303; inhibitory index 2 indicated that medium inhibitory zones were induced by the Bacillus spp. strains, with the strains with this index value including DR242, DR243, and DR244; and inhibitory index 3 indicated strong inhibitory zones were induced by the Bacillus spp. strains, with the strains with this index value including DR238 and DR301 (Table 1).
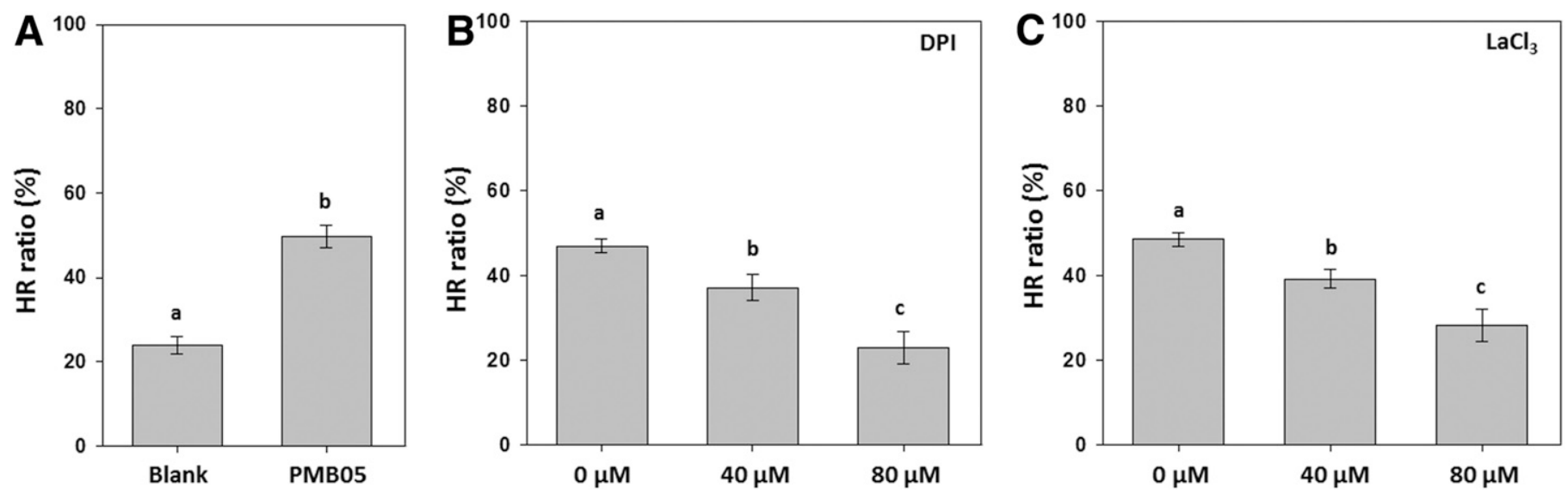

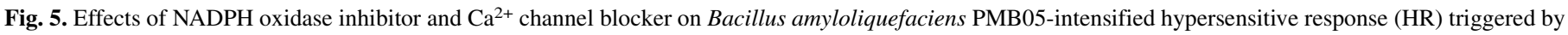

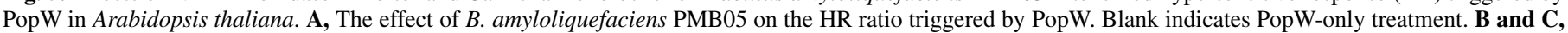

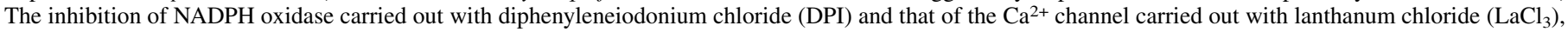

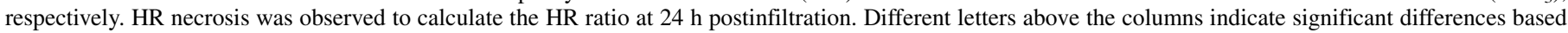

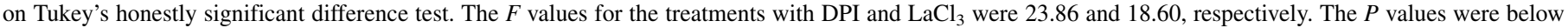
0.05 in both assays. 


\section{DISCUSSION}

The disease resistance conferred by PFLP constitutes a successful approach for protecting plants against various bacterial diseases (Ger et al. 2014; Huang et al. 2007; Liau et al. 2003; Lin et al. 2011; Lin et al. 2010; Namukwaya et al. 2012; Tang et al. 2001; Yip et al. 2007). This enhanced disease resistance is associated with the activation of flagellin (flg22) mediated PTI (Hong et al. 2018; Su et al. 2014). Due to concerns regarding the application of genetically engineered organisms, alternative strategies involving the use of microorganisms to intensify the innate immunity of plants have been viewed as promising. In this study, we sought to screen bacterial strains from rhizosphere that could intensify PTI to further confer disease resistance against
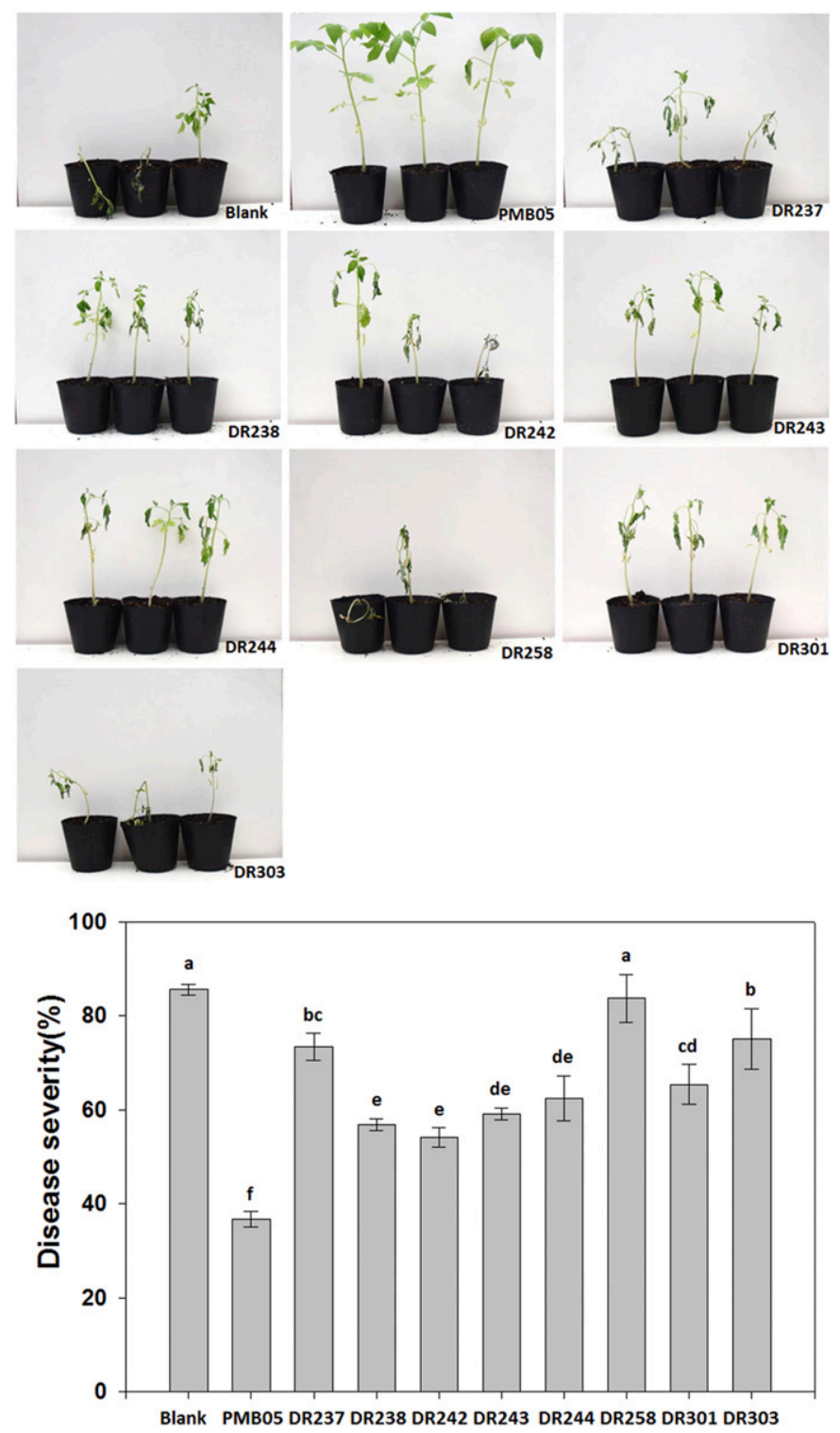

Fig. 6. Effects of Bacillus spp. strains on control of tomato bacterial wilt. Upper panel and lower panel reveal the symptom appearance and disease severity of bacterial wilt at 10 days postinoculation, respectively. The treatments with the Bacillus sp. strains were performed with 3-week-old tomato plants soaked in the bacterial suspensions before inoculation. Blank indicates that the treatment consisted of sterilized water only. Then, the plants were transplanted into diseased soil containing Ralstonia solanacearum Ps152 to evaluate the indexes of wilting symptoms at 1 week postinoculation. Different letters above the disease severity columns indicate significant differences based on Tukey's honestly significant difference test $(F=68.49 ; P<0.05)$. bacterial wilt of tomato caused by $R$. solanacearum. Given this objective, a PAMP from this pathogen was required in our assay. A harpin from $R$. solanacearum, PopW, is a PAMP that can elicit the HR in tobacco leaves ( $\mathrm{Li}$ et al. 2010). In this study, then, we expressed and purified the PopW protein from E. coli, and HR necrosis was induced in the infiltrated areas of Arabidopsis leaves postinfiltration. The occurrence of HR necrosis indicates the degree of defense amplification over the typical activation of PTI (Jones and Dangl 2006). Here, we showed that the ratios relating to HR necrosis induced by purified PopW was dose-dependent, and the concentration of this protein that elicited around $20 \% \mathrm{HR}$ necrosis was applied and regarded as the basal defense response induction. Therefore, we believe that Arabidopsis plants can offer better resolution to confirm the effects of Bacillus spp. strains on this HR reaction.

The intensification of PopW-induced HR carried out with Bacillus spp. strains was performed to screen for a possible defense signals-intensifying strain. Increased HR ratios were not observed, however, following the treatments performed with most Bacillus spp. strains. Among all the tested strains, in fact, the B. amyloliquefaciens strain PMB05 was the only strain that increased the HR ratio upon the infiltration of PopW. This result was similar to the intensification of flg22 $2_{\mathrm{Pst}}$-induced HR carried out with PFLP in previous studies (Hong et al. 2018; Su et al. 2014). Previously, we also demonstrated that the HrpN-mediated PTI signals were intensified by $B$. amyloliquefaciens strain PMB05 (Wang et al. 2019). Similar results of PTI signal intensification by strain PMB05 were observed in the treatment of PopW in this study. Furthermore, the intensification of the PopW-induced HR by PMB05 was strongly reduced when an NADPH oxidase inhibitor or calcium channel blocker was applied. Therefore, we suggest that B. amyloliquefaciens PMB05 is a bacterial strain that can intensify PTI signals to reach the threshold of HR. Recent studies have shown that plant defense responses such as ROS generation and callose deposition are activated by $B$. cereus AR156 during fungal pathogen infection and further confer disease resistance to Botrytis cinerea and Pseudomonas syringae pv. tomato in Arabidopsis (Nie et al. 2017; Niu et al. 2016). To further clarify whether PMB05 can confer resistance to tomato bacterial wilt by intensifying PTI, the evaluation of its efficacy in controlling bacterial wilt disease is critical. The results indicated that the PTI-intensifying strain, PMB05, was the most effective strain in terms of increasing disease resistance. To link such increased disease resistance in tomato plants with the PTI intensification caused by PMB05, analyses of the defense signals of tomato plants are required. Methods to study PTI responses in tomato have been well established based on P. syringae pv. tomato (Nguyen et al. 2010). However, we could not successfully analyze the gene expression

TABLE 1. Inhibitory effects of Bacillus spp. strains against Ralstonia solanacearum on nutrient agar plates ${ }^{\mathrm{z}}$

\begin{tabular}{lccc}
\hline Strain & Inhibitory zone $(\mathrm{mm})$ & Inhibitory index & PTI-intensifying \\
\hline PMB05 & $5.67 \mathrm{ab}$ & 1 & + \\
DR237 & $3.31 \mathrm{a}$ & 1 & - \\
DR238 & $17.24 \mathrm{~d}$ & 3 & - \\
DR242 & $9.91 \mathrm{c}$ & 2 & - \\
DR243 & $7.83 \mathrm{bc}$ & 2 & - \\
DR244 & $10.34 \mathrm{c}$ & 2 & - \\
DR258 & $3.27 \mathrm{a}$ & 1 & - \\
DR301 & $15.92 \mathrm{~d}$ & 3 & - \\
DR303 & $5.21 \mathrm{ab}$ & 1 & - \\
\hline
\end{tabular}

$\mathrm{z}$ The inhibitory zone was calculated as follows: the outer diameter of the antagonist circle - the diameter of the antagonistic colony/2. Letters after the inhibitory zone mean values indicate significant differences based on Tukey's honestly significant difference test $(F=90.09 ; P<0.05)$. The inhibitory index values 1,2 , and 3 indicate that the inhibitory zones of the Bacillus spp. strains against $R$. solanacearum were in the range of $<6,6$ to 12 , and $>12 \mathrm{~mm}$, respectively. PTI, PAMP-triggered immunity. 
profiles by using specific primers designed by genes such as Pti5 or Lrr22 in the quantitative RT-PCR. We speculated that this might have been due to the variability in the gene sequences of the tomato varieties we used in this study. In addition, we used a leaf infiltration assay to evaluate whether the PTI intensification would cause a reduction of such infiltration by $R$. solanacearum on tomato leaves. The results indicated only a slight reduction in the bacterial population of $R$. solanacearum on leaves at 2 days postinfiltration (Supplementary Fig. S1), but the population was uncountable at 4 days postinfiltration. This led us to speculate that increasing population levels of some environmental microorganisms during HR may affect the counting of $R$. solanacearum on the SM1 selective medium. Even so, this study also used different PAMPs to trigger PTI signals on tomato leaves, and further proved that treatment with PMB05 can indeed strengthen these defense responses. Furthermore, we found that the reduction of tomato bacterial wilt disease severity caused by most of the Bacillus spp. strains was associated with the strength of their antagonistic activity. Interestingly, PMB05 is the only strain that has weak antagonistic effects but still effectively enhances resistance to tomato bacterial wilt. Therefore, we suggested that the PTI intensification caused by microbial strains may be a key factor that is more important than the antagonistic activity in the application of microorganisms to control tomato bacterial wilt. Past reports have demonstrated that plant immune responses are associated with the salicylic acid defense pathway or jasmonate/ethylene defense pathway (Eulgem and Somssich 2007; Nie et al. 2016, 2017; Pieterse et al. 2009; Torres 2010). The associations of these pathways with the PopW-induced signals induced by PMB05, however, are still unknown and need to be further investigated.

Taken together, our results led us to conclude that the disease resistance against tomato bacterial wilt enhanced by $B$. amyloliquefaciens PMB05 is associated with its intensification of PTI-triggered defense responses. Most importantly, the results in this study demonstrate that the PTI intensified by Bacillus spp. might play a key role in enhancing disease resistance. It is thus suggested that screening for PTI-intensifying Bacillus spp. strains to use as biocontrol agents to control plant diseases could be a promising strategy for plant protection in the future.

\section{ACKNOWLEDGMENTS}

We thank Li-Hsin Wu for providing helpful comments on the statistical analysis in this manuscript.
A
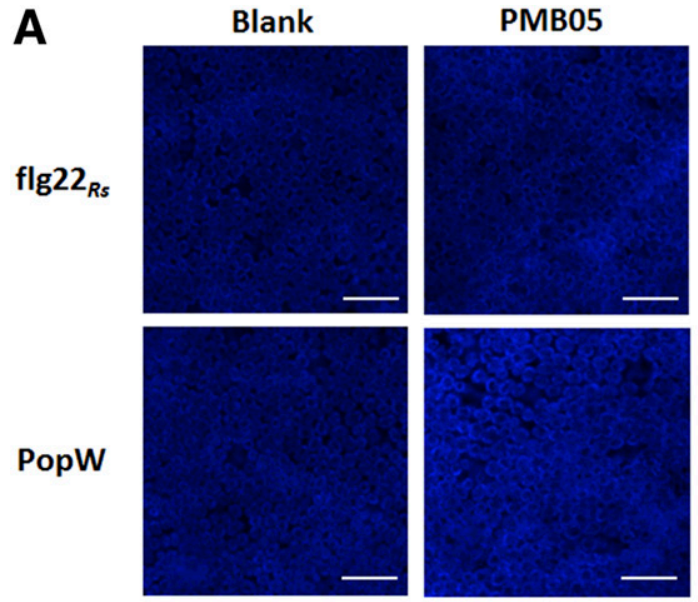

B

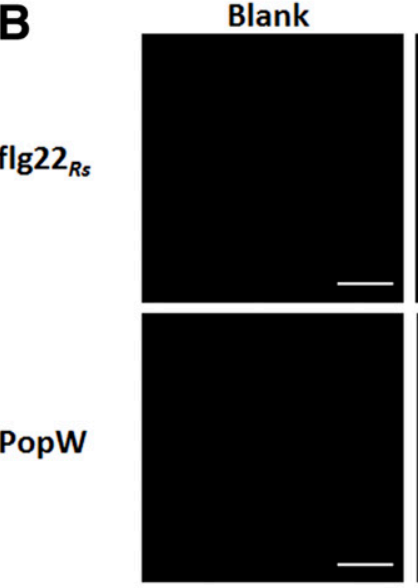

PMB05
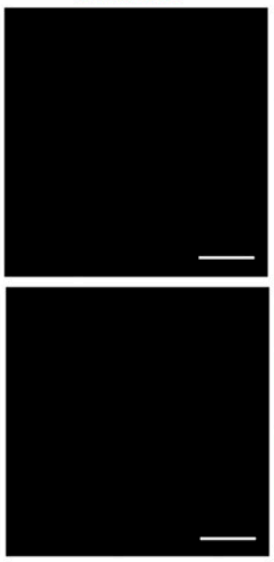

PAMP
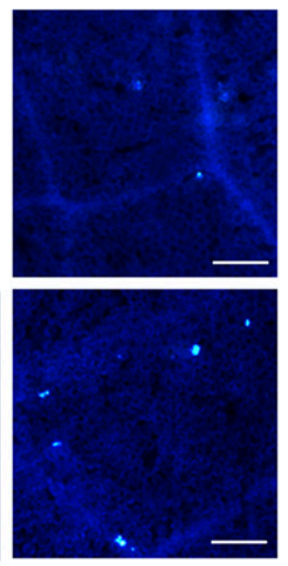

PAMP
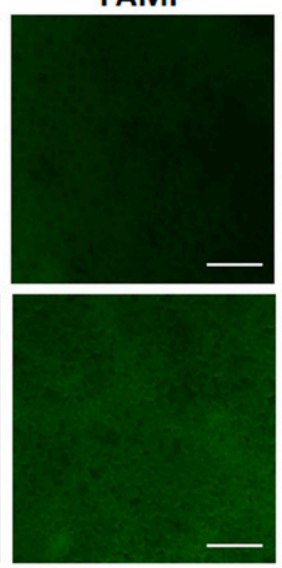

PAMP/PMB05

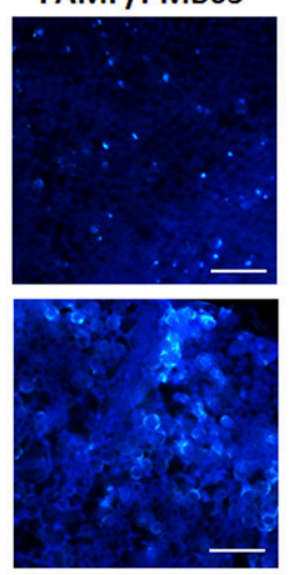

PAMP/PMB05
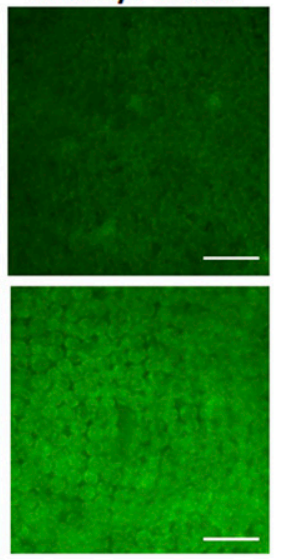
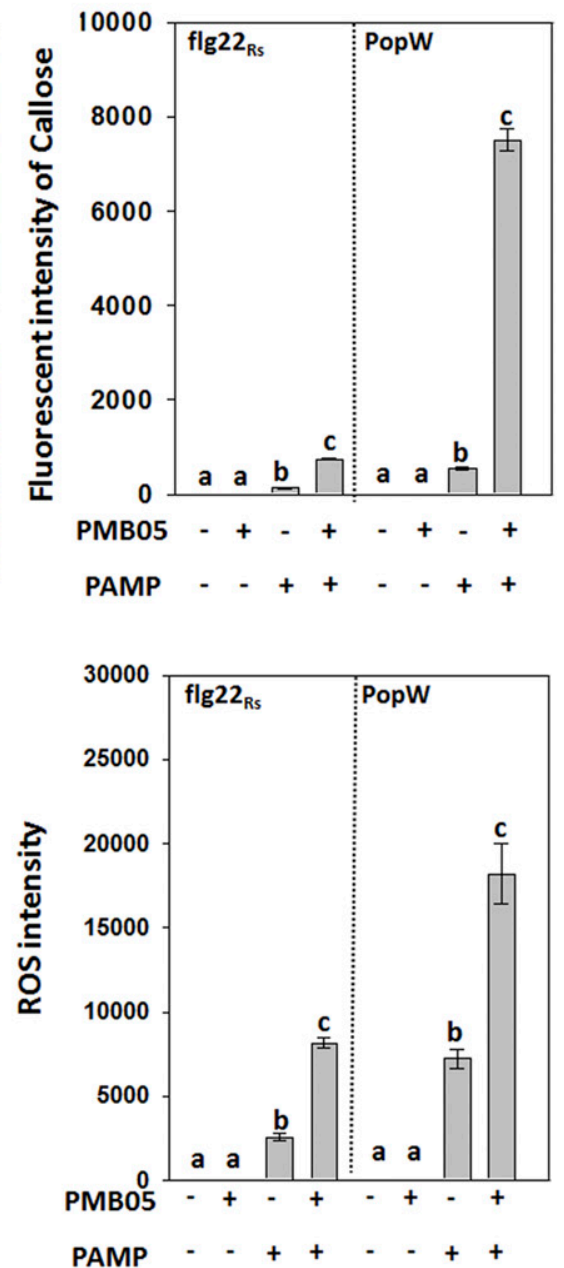

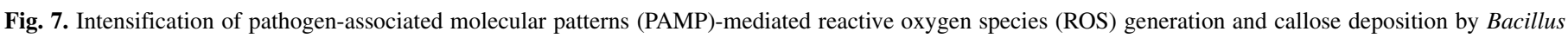

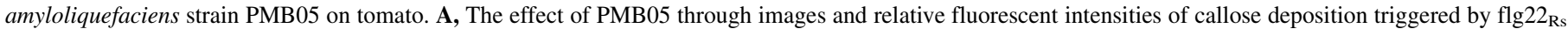

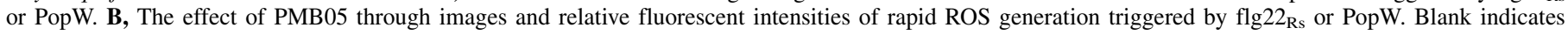

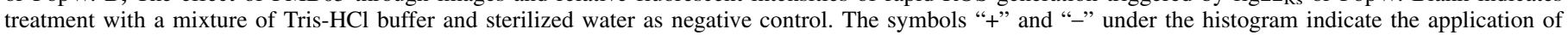

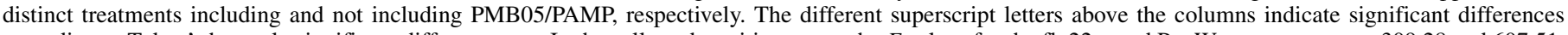

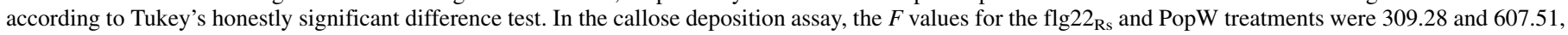

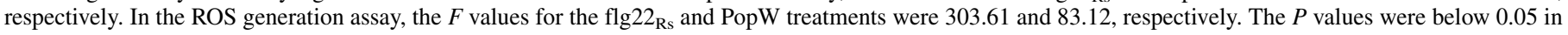
all assays. 


\section{LITERATURE CITED}

Ali, G. S., and Reddy, A. S. N. 2008. PAMP-triggered immunity: Early events in the activation of FLAGELLIN SENSITIVE2. Plant Signal. Behav. 3: 423-426.

Bigeard, J., Colcombet, J., and Hirt, H. 2015. Signaling mechanisms in pattern-triggered immunity (PTI). Mol. Plant 8:521-539.

Boller, T., and Felix, G. 2009. A renaissance of elicitors: Perception of microbe-associated molecular patterns and danger signals by patternrecognition receptors. Annu. Rev. Plant Biol. 60:379-406.

Boudsocq, M., Willmann, M. R., McCormack, M., Lee, H., Shan, L., He, P., Bush, J., Cheng, S. H., and Sheen, J. 2010. Differential innate immune signalling via $\mathrm{Ca} 2+$ sensor protein kinases. Nature 464:418-422.

Chinchilla, D., Bauer, Z., Regenass, M., Boller, T., and Felix, G. 2006. The Arabidopsis receptor kinase FLS2 binds flg22 and determines the specificity of flagellin perception. Plant Cell 18:465-476.

de Boer, A. S., and Diderichsen, B. 1991. On the safety of Bacillus subtilis and B. amyloliquefaciens: A review. Appl. Microbiol. Biotechnol. 36:1-4.

Dodds, P. N., and Rathjen, J. P. 2010. Plant immunity: Towards an integrated view of plant-pathogen interactions. Nat. Rev. Genet. 11:539-548.

Eulgem, T., and Somssich, I. E. 2007. Networks of WRKY transcription factors in defense signaling. Curr. Opin. Plant Biol. 10:366-371.

Genin, S. 2010. Molecular traits controlling host range and adaptation to plants in Ralstonia solanacearum. New Phytol. 187:920-928.

Ger, M.-J., Louh, G.-Y., Lin, Y.-H., Feng, T.-Y., and Huang, H.-E. 2014. Ectopic expressed sweet pepper ferredoxin PFLP enhances disease resistance to Pectobacterium carotovorum subsp. carotovorum affected by harpin and protease-mediated hypersensitive response in Arabidopsis. Mol. Plant Pathol. 15:892-906.

Grey, B. E., and Steck, T. R. 2001. The viable but nonculturable state of Ralstonia solanacearum may be involved in long-term survival and plant infection. Appl. Environ. Microbiol. 67:3866-3872.

Guan, X., Buchholz, G., and Nick, P. 2013. The cytoskeleton is disrupted by the bacterial effector HrpZ, but not by the bacterial PAMP flg22, in tobacco BY-2 cells. J. Exp. Bot. 64:1805-1816.

Gust, A. A., Biswas, R., Lenz, H. D., Rauhut, T., Ranf, S., Kemmerling, B., Götz, F., Glawischnig, E., Lee, J., Felix, G., and Nürnberger, T. 2007. Bacteria-derived peptidoglycans constitute pathogen-associated molecular patterns triggering innate immunity in Arabidopsis. J. Biol. Chem. 282: $32338-32348$

Hayward, A. C. 1991. Biology and epidemiology of bacterial wilt caused by Pseudomonas solanacearum. Annu. Rev. Phytopathol. 29:65-87.

Hong, C.-Y., Zheng, J.-L., Chen, T.-Y., Chao, H.-R., and Lin, Y.-H. 2018. PFLPintensified disease resistance against bacterial soft rot through MAPK pathway in PAMP-triggered immunity. Phytopathology 108:1467-1474.

Huang, C.-N., Lin, C.-P., Hsieh, F.-C., Lee, S.-K., Cheng, K.-C., and Liu, C.-T. 2016. Characterization and evaluation of Bacillus amyloliquefaciens strain WF02 regarding its biocontrol activities and genetic responses against bacterial wilt in two different resistant tomato cultivars. World J. Microbiol. Biotechnol. 32:183.

Huang, H.-E., Liu, C.-A., Lee, M.-J., Kuo, C.-G., Chen, H.-M., Ger, M.-J., Tsai, Y.-C., Chen, Y.-R., Lin, M.-K., and Feng, T.-Y. 2007. Resistance enhancement of transgenic tomato to bacterial pathogens by the heterologous expression of sweet pepper ferredoxin-I protein. Phytopathology 97: 900-906.

Jones, J. D. G., and Dangl, J. L. 2006. The plant immune system. Nature 444: 323-329.

Kelman, A. 1954. The relationship of pathogenicity in Pseudomonas solanacearum to colony appearance on a tetrazolium medium. Phytopathology 44:693-695.

Kumar, P., Dubey, R. C., and Maheshwari, D. K. 2012. Bacillus strains isolated from rhizosphere showed plant growth promoting and antagonistic activity against phytopathogens. Microbiol. Res. 167:493-499.

Kwon, J. W., and Kim, S. D. 2014. Characterization of an antibiotic produced by Bacillus subtilis JW-1 that suppresses Ralstonia solanacearum. J. Microbiol. Biotechnol. 24:13-18.

Li, J.-G., Cao, J., Sun, F.-F., Niu, D.-D., Yang, F., Liu, H.-X., and Guo, J.-H. 2011. Control of Tobacco mosaic virus by PopW as a result of induced resistance in tobacco under greenhouse and field conditions. Phytopathology 101:1202-1208.

Li, J.-G., Liu, H.-X., Cao, J., Chen, L.-F., Gu, C., Allen, C., and Guo, J.-H. 2010. PopW of Ralstonia solanacearum, a new two-domain harpin targeting the plant cell wall. Mol. Plant Pathol. 11:371-381.

Liau, C.-H., Lu, J.-C., Prasad, V., Hsiao, H.-H., You, S.-J., Lee, J.-T., Yang, N.-S., Huang, H.-E., Feng, T.-Y., Chen, W.-H., and Chan, M.-T. 2003. The sweet pepper ferredoxin-like protein ( $p f l p)$ conferred resistance against soft rot disease in Oncidium orchid. Transgenic Res. 12:329-336.

Lin, Y.-H., Huang, H.-E., Chen, Y.-R., Liao, P.-L., Chen, C.-L., and Feng, T.-Y. 2011. C-terminal region of plant ferredoxin-like protein is required to enhance resistance to bacterial disease in Arabidopsis thaliana. Phytopathology 101:741-749.

Lin, Y.-H., Huang, H.-E., Wu, F.-S., Ger, M.-J., Liao, P.-L., Chen, Y.-R., Tzeng, K.-C., and Feng, T.-Y. 2010. Plant ferredoxin-like protein (PFLP) outside chloroplast in Arabidopsis enhances disease resistance against bacterial pathogens. Plant Sci. 179:450-458.

Liu, H., Wang, Y., Zhou, X., Wang, C., Wang, C., Fu, J., and Wei, T. 2016. Overexpression of a harpin-encoding gene popW from Ralstonia solanacearum primed antioxidant defenses with enhanced drought tolerance in tobacco plants. Plant Cell Rep. 35:1333-1344.

Monaghan, J., and Zipfel, C. 2012. Plant pattern recognition receptor complexes at the plasma membrane. Curr. Opin. Plant Biol. 15:349-357.

Namukwaya, B., Tripathi, L., Tripathi, J. N., Arinaitwe, G., Mukasa, S. B., and Tushemereirwe, W. K. 2012. Transgenic banana expressing pflp gene confers enhanced resistance to Xanthomonas wilt disease. Transgenic Res. 21:855-865.

Nguyen, H. P., Chakravarthy, S., Velásquez, A. C., McLane, H. L., Zeng, L., Nakayashiki, H., Park, D.-H., Collmer, A., and Martin, G. B. 2010. Methods to study PAMP-triggered immunity using tomato and Nicotiana benthamiana. Mol. Plant-Microbe Interact. 23:991-999.

Nie, P., Li, X., Wang, S., Guo, J., Zhao, H., and Niu, D. 2017. Induced systemic resistance against Botrytis cinerea by Bacillus cereus AR156 through a JA/ET- and NPRI-dependent signaling pathway and activates PAMP-triggered immunity in Arabidopsis. Front. Plant Sci. 8:238.

Niu, D., Wang, X., Wang, Y., Song, X., Wang, J., Guo, J., and Zhao, H. 2016. Bacillus cereus AR156 activates PAMP-triggered immunity and induces a systemic acquired resistance through a NPRI- and SA-dependent signaling pathway. Biochem. Biophys. Res. Commun. 469:120-125.

Oh, C.-S., and Beer, S. V. 2005. Molecular genetics of Erwinia amylovora involved in the development of fire blight. FEMS Microbiol. Lett. 253: 185-192.

Pieterse, C. M. J., Leon-Reyes, A., Van der Ent, S., and Van Wees, S. C. M. 2009. Networking by small-molecule hormones in plant immunity. Nat. Chem. Biol. 5:308-316.

Postel, S., and Kemmerling, B. 2009. Plant systems for recognition of pathogen-associated molecular patterns. Semin. Cell Dev. Biol. 20: 1025-1031.

Ramadasappa, S., Rai, A. K., Jaat, R. S., Singh, A., and Rai, R. 2012. Isolation and screening of $p h l D^{+}$plant growth promoting rhizobacteria antagonistic to Ralstonia solanacearum. World J. Microbiol. Biotechnol. 28:1681-1690.

Rasband, W. S. 1997-2016. ImageJ. U.S. National Institutes of Health, Bethesda, MD. https://imagej.nih.gov/ij/

Romero, D., de Vicente, A., Rakotoaly, R. H., Dufour, S. E., Veening, J.-W., Arrebola, E., Cazorla, F. M., Kuipers, O. P., Paquot, M., and Pérez-García, A. 2007. The iturin and fengycin families of lipopeptides are key factors in antagonism of Bacillus subtilis toward Podosphaera fusca. Mol. PlantMicrobe Interact. 20:430-440.

Song, S., Fu, S., Sun, X., Li, P., Wu, J., Dong, T., He, F., and Deng, Y. 2018. Identification of cyclic dipeptides from Escherichia coli as new antimicrobial agents against Ralstonia solanacearum. Molecules 23:214.

Su, Y.-H., Hong, C.-Y., and Lin, Y.-H. 2014. Plant ferredoxin-like protein enhances resistance to bacterial soft rot disease through PAMPtriggered immunity in Arabidopsis thaliana. Eur. J. Plant Pathol. 140: 377-384.

Tahir, H. A., Gu, Q., Wu, H., Niu, Y., Huo, R., and Gao, X. 2017. Bacillus volatiles adversely affect the physiology and ultra-structure of Ralstonia solanacearum and induce systemic resistance in tobacco against bacterial wilt. Sci. Rep. 7:40481.

Tang, K. X., Sun, X. F., Hu, Q. N., Wu, A. Z., Lin, C. H., Lin, H.-J., Twyman, R. M., Christou, P., and Feng, T.-Y. 2001. Transgenic rice plants expressing the ferredoxin-like protein (AP1) from sweet pepper show enhanced resistance to Xanthomonas oryzae pv. oryzae. Plant Sci. 160:1035-1042.

Torres, M. A. 2010. ROS in biotic interactions. Physiol. Plant. 138:414-429. van Elsas, J. D., Kastelein, P., van Bekkum, P., van der Wolf, J. M., de Vries, P. M., and van Overbeek, L. S. 2000. Survival of Ralstonia solanacearum biovar 2, the causative agent of potato brown rot, in field and microcosm soils in temperate climates. Phytopathology 90:1358-1366.

Wang, Y.-H., Lai, I.-L., Zheng, J.-L., and Lin, Y.-H. 2019. Using dynamic changes of chlorophyll fluorescence in Arabidopsis thaliana to evaluate plant immunity-intensifying Bacillus spp. strains. Phytopathology 109: 1566-1576.

Winstead, N. N., and Kelman, A. 1952. Inoculation techniques for evaluating resistance to Pseudomonas solanacearum. Phytopathology 42:628-634.

Yabuuchi, E., Kosako, Y., Yano, I., Hotta, H., and Nishiuchi, Y. 1995. Transfer of two Burkholderia and an Alcaligenes species to Ralstonia gen. Nov.: Proposal of Ralstonia pickettii (Ralston, Palleroni and Doudoroff 1973) comb. Nov., Ralstonia solanacearum (Smith 1896) comb. Nov. and Ralstonia eutropha (Davis 1969) comb. Nov. Microbiol. Immunol. 39: 894-904. 
Yim, W., Seshadri, S., Kim, K., Lee, G., and Sa, T. 2013. Ethylene emission and PR protein synthesis in ACC deaminase producing Methylobacterium spp. inoculated tomato plants (Lycopersicon esculentum Mill.) challenged with Ralstonia solanacearum under greenhouse conditions. Plant Physiol. Biochem. 67:95-104.

Yip, M.-K., Huang, H.-E., Ger, M.-J., Chiu, S.-H., Tsai, Y.-C., Lin, C.-I., and Feng, T.-Y. 2007. Production of soft rot resistant calla lily by expressing a ferredoxinlike protein gene $(p f l p)$ in transgenic plants. Plant Cell Rep. 26:449-457.
Yip, M.-K., Lee, S.-W., Su, K.-C., Lin, Y.-H., Chen, T.-Y., and Feng, T. Y. 2011. An easy and efficient protocol in the production of pflp transgenic banana against Fusarium wilt. Plant Biotechnol. Rep. 5: 245-254.

Zalila-Kolsi, I., Mahmoud, A. B., Ali, H., Sellami, S., Nasfi, Z., Tounsi, S., and Jamoussi, K. 2016. Antagonist effects of Bacillus spp. strains against Fusarium graminearum for protection of durum wheat (Triticum turgidum L. subsp. durum). Microbiol. Res. 192:148-158. 